\title{
Lattice simulation of lower dimensional SYM with sixteen supercharges
}

\author{
Daisuke Kadoh* \\ High Energy Accelerator Research Organization (KEK), Tsukuba 305-0801, Japan \\ E-mail: kadoh@post.kek.jp
}

\section{Syo Kamata}

Department of Physics, Rikkyo University, Tokyo 171-8501, Japan

E-mail: skamata@rikkyo.ac.jp

\begin{abstract}
We report on the lattice simulation result of one dimensional supersymmetric Yang-Mills theory with sixteen supercharges. According to the gauge/gravity duality, this theory is expected to be dual to $N$ D0-branes in type IIA superstring/supergravity. If the imaginary time direction is interpreted as temperature, in the large $N$ limit, low temperature region where the gauge theory is strongly coupled corresponds to a classical black hole. We examine the gauge theory side by the lattice simulation to verify the duality conjecture. In this article, we focus on the internal energy of the black hole. The internal energy obtained by the simulation is compared with high temperature expansion at high temperatures, on the other hand, it is getting close to the analytic solution of the gravity side at low temperatures.
\end{abstract}

31st International Symposium on Lattice Field Theory - LATTICE 2013

July 29 - August 3, 2013

Mainz, Germany

\footnotetext{
*Speaker.
} 


\section{Introduction}

Gauge/gravity duality has become one of the most exciting topics in particle physics. If it is correct, physical quantities in strongly coupled gauge theory can be solved exactly from the gravity side, and conversely, unknown properties of string theory can be understood from the gauge theory side. Despite such virtues, it is however a conjecture, and giving some kind of rigorous proof is still an important subject. In this article, we perform a lattice simulation of one dimensional supersymmetric Yang-Mills theory with sixteen supercharges (the BFSS model), which is expected to be dual to $N$ D0-branes in type IIA superstring/supergravity. This duality is a low dimensional version of the AdS/CFT correspondence[1], but the theory has no conformal symmetry. We examine the duality conjecture by comparing lattice results with analytic solutions of the gravity side.

A few numerical simulations for this theory has been already done. Nishimura et al. used the theory regularized by the momentum sharp cut-off and obtained results that suggest the validity of the duality including $\alpha^{\prime}$ corrections [2,3,4,5]. Also, Catterall and Wiseman performed lattice simulations[6], and their results did not indicate apparent contradictions of the duality within somewhat large statistical errors at low temperatures. But both results have not yet explained the leading-order temperature dependence of the internal energy of the black hole within a few percent statistical errors, which is expected from the gravity side in the large $N$ limit.

We aim to test the gauge/gravity duality with a high degree of accuracy by employing a lattice action with two exact supercharges. In this article, we focus on the internal energy of the black hole as one of typical physical quantities in the gravity side. At high temperatures, the energy obtained by the simulation shows good agreement with the high temperature expansion of the gauge theory, and, at low temperatures, it is getting close to the analytic solution of the gravity side in the large $N$ limit.

This paper is organized as follows. In section 2, we briefly review the continuum theory and the lattice theory. Some simulation techniques are described in section 3. Then, we explain the high temperature expansion of the gauge theory in section 4. Finally, we show simulation results of the black hole internal energy in section 5 .

\section{Continuum and lattice action}

Supersymmetric Yang-Mills theory in $\mathrm{d}=1+0$ with sixteen supercharges contains a temporal gauge field $A_{0}$, nine adjoint scalars $X_{i}(i=1, \cdots, 9)$, and sixteen adjoint fermions $\psi_{\alpha}(\alpha=$ $1, \cdots, 16)$. In Euclidean time with period $\beta$, the action is given by

$$
\begin{aligned}
S_{\text {cont. }}=\frac{N}{\lambda} \int_{0}^{\beta} \mathrm{d} t \operatorname{tr}\left\{\frac{1}{2}\left(D_{0} X_{i}\right)^{2}-\frac{1}{4}\left[X_{i}, X_{j}\right]^{2}\right. \\
\left.+\frac{1}{2} \psi_{\alpha} D_{0} \psi_{\alpha}+\frac{1}{2} \psi_{\alpha}\left(\gamma_{i}\right)_{\alpha \beta}\left[X_{i}, \psi_{\beta}\right]\right\},
\end{aligned}
$$

where $\lambda$ is the 't Hooft coupling constant and $\gamma_{i}$ are real symmetric matrices which satisfy Clifford algebra for nine dimensional Euclidean space, $\left\{\gamma_{i}, \gamma_{j}\right\}=2 \delta_{i j}$. Also, the gauge field has no kinetic terms and interacts with the other fields only through a covariant derivative $D_{0}$ which is defined as $D_{0} \varphi=\partial_{t} \varphi+i\left[A_{0}, \varphi\right]$ for adjoint fields $\varphi$. 
If both bosons and fermions satisfy the periodic boundary condition for the time direction, this theory is invariant under the following supersymmetry transformation,

$$
\begin{aligned}
& \delta_{\xi} A_{0}=\xi_{\alpha} \psi_{\alpha}, \\
& \delta_{\xi} X_{i}=-i \xi_{\alpha}\left(\gamma_{i}\right)_{\alpha \beta} \psi_{\beta}, \\
& \delta_{\xi} \psi_{\alpha}=i\left(\gamma_{i}\right)_{\alpha \beta} \xi_{\beta} D_{0} X_{i}-\frac{1}{2}\left(\Sigma_{i j}\right)_{\alpha \beta} \xi_{\beta}\left[X_{i}, X_{j}\right], \quad \Sigma_{i j}=\frac{i}{2}\left[\gamma_{i}, \gamma_{j}\right],
\end{aligned}
$$

where $\xi_{\alpha}$ are fermionic global parameters. But, we impose the anti-periodic boundary condition on the fermions. So, this theory is actually a finite temperature theory in which supersymmetry is broken by temperature.

For a long time, it has been difficult to realize supersymmetry, like eq.(2.2), on the lattice because of a well-known breakdown of the Leibniz rule. However, lattice formulations that keep a few supercharges exactly on the lattice were proposed nearly a decade ago[7, 8]. In this research, we employ a lattice action with two exact supercharges, proposed by Sugino[8]. His basic idea is very simple such that the continuum action can be rewritten as an exact form of two nilpotent supercharges $Q_{ \pm}$, then the $Q_{ \pm}$-invariance are manifest from the nilpotency without the Leibniz rule, and therefore the $Q_{ \pm}$-charges can be realized even on the lattice ${ }^{1}$.

Let us consider a lattice theory on one dimensional lattice with lattice size $N_{t}$. Gauge field is defined on links as a link field $U_{0} \in S U(N)$, and the other adjoint fields are defined on sites labeled by integers $t=0, \cdots, N_{t}-1$ (a lattice spacing $a$ is set to unity without loss of generality.). After renaming fields, the continuum action eq.(2.1) can be rewritten as a $Q_{ \pm}$-exact form, and then we introduce a formally-defined lattice action from the exact one as

$$
\begin{gathered}
S=Q_{+} Q_{-} \frac{N}{2 \lambda_{0}} \sum_{t=0}^{N_{t}-1} \operatorname{tr}\left[-2 i B_{i}\left(F_{i 3}+\frac{1}{2} \varepsilon_{i j k} F_{j k}\right)-\frac{1}{3} \varepsilon_{i j k} B_{i}\left[B_{j}, B_{k}\right]\right. \\
\left.-\psi_{+\mu} \psi_{-\mu}-\chi_{+i} \chi_{-i}-\frac{1}{4} \eta_{+} \eta_{-}\right]
\end{gathered}
$$

where $\lambda_{0}$ is the dimensionless 't Hooft coupling constant and $F_{\mu \nu}$ is a field tensor, $F_{0 i}=\nabla_{0} A_{i}, F_{i j}=$ $i\left[A_{i}, A_{j}\right],(i, j=1,2,3)$, which is given by reducing dimensions from that of four dimensions. Here $\nabla_{0}$ is a covariant forward difference operator ${ }^{2}$.

$Q_{ \pm}$transformations can be given on the lattice as a closed form $[8,11]$, and satisfy

$$
Q_{ \pm}^{2}=i \delta_{\phi_{ \pm}}, \quad\left\{Q_{+}, Q_{-}\right\}=i \delta_{C},
$$

where $\delta_{\omega}$ is a lattice gauge transformation with gauge parameter $\omega$,

$$
\delta_{\omega} U(t)=-i \nabla_{0} \omega(t) U(t), \quad \delta_{\omega} \varphi(t)=-i[\varphi(t), \omega(t)],
$$

for adjoint fields $\varphi$. Thus, at least, two supercharges are realized on the lattice, that is, they are broken by temperature but not by the cut-off. Remaining fourteen charges are, however, broken by both the cut-off and temperature.

In [11], we examined the supersymmetric Ward-Takahashi identity numerically, and we have checked that all sixteen supercharges including the other fourteen charges are restored in the continuum limit in the sense that SUSY breaking cut-off effects vanish.

\footnotetext{
${ }^{1}$ In the other articles[7], more charges are realized on the lattice.

${ }^{2}$ See also $[8,11]$.
} 


\section{High temperature expansion}

At high temperatures $T \gg 1$, simulation results can be compared to a high temperature expansion of the gauge theory ${ }^{3}$. In this section, the internal energy of the black hole is presented in the high temperature expansion up to next to leading order of inverse temperature. Mathematical techniques of the expansion have already given in [9], but we found that the previous calculations for some expansion coefficients were incorrect. In the following, the results are given with corrected coefficients.

Using the Fourier transformation, the action eq.(2.1) can be expressed as a function of zero modes $A_{\mu}(\mu=0, \cdots, 9)$ and the other non-zero modes. After rescaling these field variables, interaction terms become higher order of the inverse temperature. Then the integral of non-zero modes can be treated like a perturbation theory around the high temperature limit, on the other hand, because the kinetic terms are absent, the integral of zero modes remains as expectation values of a matrix model,

$$
\langle\langle\mathscr{O}\rangle\rangle=\frac{1}{Z_{0}} \int \prod_{a, \mu} d A_{\mu}^{a} \mathscr{O} e^{\frac{N}{4} \operatorname{tr}\left(\left[A_{\mu}, A_{\nu}\right]^{2}\right)},
$$

where $Z_{0}$ the partition function of the matrix model. Generally, the expectation values can not be analytically calculated, and the expansion coefficients are determined by numerical simulations of the matrix model.

In [9], it has shown that, at the next to the leading order, the internal energy of the black hole can be given by,

$$
\langle E\rangle=6\left(1-\frac{1}{N^{2}}\right) T+\left(\chi_{5}-\chi_{6}-4 \chi_{1}\right) T^{-1 / 2}+\mathscr{O}\left(T^{-2}\right),
$$

where $\chi_{1}, \chi_{5}, \chi_{6}$ are given by

$$
\begin{aligned}
& \chi_{1}=\frac{9}{10}\left\langle\left\langle\operatorname{tr}\left(A_{\mu}^{2}\right)\right\rangle\right\rangle, \\
& \chi_{5}=\left\langle\left\langle\operatorname{tr}\left(\left[A_{i}, A_{j}\right]^{2}\right) \operatorname{tr}\left(A_{k}^{2}\right)\right\rangle\right\rangle-\left\langle\left\langle\operatorname{tr}\left(\left[A_{i}, A_{j}\right]^{2}\right)\right\rangle\right\rangle\left\langle\left\langle\operatorname{tr}\left(A_{k}^{2}\right)\right\rangle\right\rangle, \\
& \chi_{6}=\left\langle\left\langle\operatorname{tr}\left(\left[A_{i}, A_{j}\right]^{2}\right) \operatorname{tr}\left(A_{0}^{2}\right)\right\rangle\right\rangle-\left\langle\left\langle\operatorname{tr}\left(\left[A_{i}, A_{j}\right]^{2}\right)\right\rangle\right\rangle\left\langle\left\langle\operatorname{tr}\left(A_{0}^{2}\right)\right\rangle\right\rangle,
\end{aligned}
$$

where $i, j, k=1, \cdots 9$ and $\mu=0,1, \cdots, 9$.

Table 1 shows coefficients that are obtained by numerical simulations of the matrix model. The previous result by Kawahara-Nishimura-Takeuchi is shown in the upper row, and the result of our re-calculation is shown in the lower row. The values of $\chi_{1}$ are consistent between two results, but those of $\chi_{5}$ and $\chi_{6}$ are different beyond statistical errors.

To see where the differences come from, let us consider Schwinger-Dyson equations,

$$
\begin{aligned}
& \sum_{a} \int D A \frac{\partial}{\partial A_{0}^{a}}\left\{A_{0}^{a} \operatorname{tr}\left(A_{0}^{2}\right) e^{\frac{N}{4} \operatorname{tr}\left(\left[A_{\mu}, A_{v}\right]^{2}\right)}\right\}=0 \\
& \sum_{a, i} \int D A \frac{\partial}{\partial A_{i}^{a}}\left\{A_{i}^{a} \operatorname{tr}\left(A_{0}^{2}\right) e^{\frac{N}{4} \operatorname{tr}\left(\left[A_{\mu}, A_{v}\right]^{2}\right)}\right\}=0 .
\end{aligned}
$$

\footnotetext{
${ }^{3}$ In this section, $\lambda$ is set to unity for simplicity, and dimensionless $T$ is used instead of $T / \lambda^{1 / 3}$.
} 
Table 1: Simulation results of the matrix model in $\mathrm{N}=32$. The upper row shows the Kawahara-NishimuraTakeuchi's previous result, and the lower row shows the re-simulation result of this work.

\begin{tabular}{l|ccccc}
\hline & $\chi_{1}$ & $\chi_{5}$ & $\chi_{6}$ & $\frac{\chi_{5}}{2 \chi_{1}}$ & $-\frac{9 \chi_{6}}{2 \chi_{1}}$ \\
\hline KNT & $2.29566(7)$ & $4.38(2)$ & $-0.59(1)$ & $0.954(4)$ & $1.157(20)$ \\
this work & $2.29573(9)$ & $4.5913(1)$ & $-0.5101(0)$ & $0.998(2)$ & $1.000(2)$ \\
\hline
\end{tabular}

These equations tell us that three coefficients are not actually independent each other,

$$
\chi_{5}=2 \chi_{1}, \quad \chi_{6}=-\frac{2}{9} \chi_{1},
$$

and then the internal energy can be given only by $\chi_{1}$,

$$
\langle E\rangle=6\left(1-\frac{1}{N^{2}}\right) T-\frac{16}{9} \chi_{1} T^{-1 / 2}+\mathscr{O}\left(T^{-2}\right) .
$$

The table 1 also shows the ratios eq.(3.8) calculated from both results. The ratios indicate that the SD equations are inconsistent with the previous result, but consistent with our re-calculation. So we concluded that there would be some mistakes in the previous calculations of the coefficients, at least, for $\chi_{5}$ and $\chi_{6}$. In section 5 , we show a lattice simulation result of the internal energy, with eq.(3.9) after use of the SD equations rather than eq.(3.2).

\section{Simulation details}

We use the hybrid Monte Carlo sampling. The lattice action has a cut-off order four fermi interaction at finite lattice spacing. By introducing a auxiliary field $\sigma$, We express the four fermi interaction as a Yukawa interaction,

$$
-\frac{1}{4} \sum_{t} \operatorname{tr}\left(\left\{\psi_{+0}, \psi_{-0}\right\}^{2}\right) \rightarrow \frac{1}{2} \sum_{t} \operatorname{tr}\left(\sigma^{2}-\frac{1}{2} \sigma\left\{\psi_{+0}, \psi_{-0}\right\}\right) .
$$

Therefore, configurations generated by HMC consist of eleven bosonic fields, $U_{0}, X_{1}, \cdots, X_{9}$, and the additional $\sigma$. Dynamical fermion effect is included through the pseudo fermion method that is applied to the absolute value of the fermion pfaffian, but the remaining phase is simply quenched.

At high temperatures, sufficient statistics were obtained by stable HMC runs. But, at low temperatures, magnitudes of the scalar fields were monotonically increasing among HMC runs, and therefore the thermalization did not occur. This instability of HMC is related to flat directions of the classical action. Actually, a temperature that begins to run away to the flat directions depended strongly on $N$, and the temperature of larger $N$ tended to be lower. The instability were not observed at temperatures higher than $T \simeq 0.55$ for $N=14$ and $T \simeq 3.5$ for $N=32$, for lattice sizes $N_{t}=8,16$. So, results in next section are limited to such parameter regions.

\section{Internal energy of black hole}

The internal energy of the black hole would be one of typical quantities to check the validity of the duality conjecture. In the gravity side, it can be given as the following analytic function of 

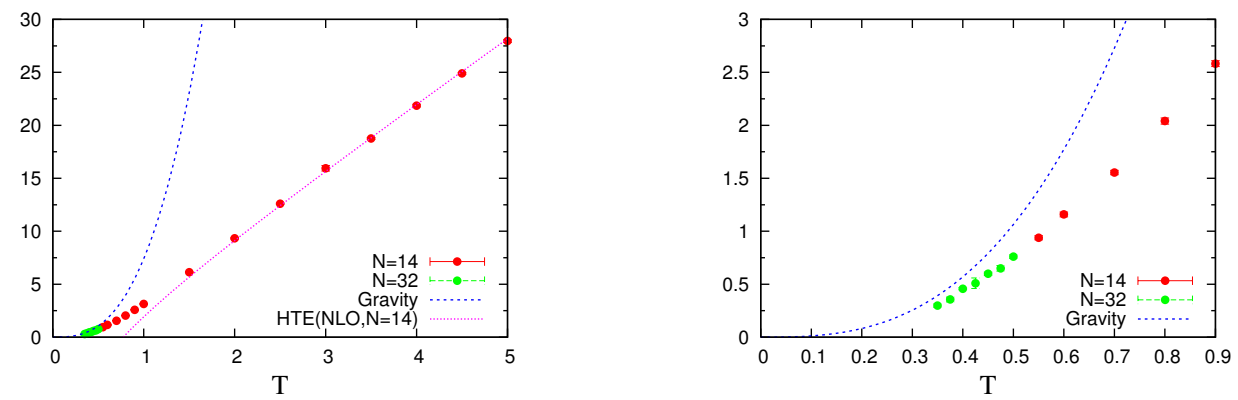

Figure 1: The internal energy of the black hole, normalized by $N^{2}$. Data points denote our results for $N=14$ (red circles) and for $N=32$ (green circles). The dashed red curve corresponds to the result obtained by the high temperature expansion up to next to leading order at $N=14$. The dashed blue curve represents the analytic solution of the gravity side in the large $N$ limit.

temperature with a coefficient $c_{1}$ in the large $N \operatorname{limit}[10]$,

$$
\frac{1}{N^{2}}\left(\frac{E}{\lambda^{1 / 3}}\right)=c_{1}\left(\frac{T}{\lambda^{1 / 3}}\right)^{14 / 5}, \quad c_{1}=\frac{9}{14}\left\{4^{13} 15^{2}\left(\frac{\pi}{7}\right)^{14}\right\}^{1 / 5}=7.41 \cdots
$$

While, in the gauge theory side, it can be determined by measuring an expectation value of the action[6],

$$
\langle E\rangle=-3 T\left\{\left\langle S_{B}\right\rangle-\frac{9}{2} N_{t}\left(N_{c}^{2}-1\right)\right\}
$$

The last term is a sum of contributions from the integral of sixteen fermions and those of seven auxiliary fields setting an origin of the energy to zero. Actually, we used -5 as a coefficient of the last term instead of -4.5 , because the auxiliary field $\sigma$ remains in the boson action and its integral yields 0.5 .

We previously reported that the internal energy obtained by the simulation in $N=14$ was getting close to the expectation of the gravity side as temperature decreases up to $T=5.5$, but $\mathrm{N}$ had to be changed to a larger one to proceed further simulations at lower temperatures. This time, we performed a simulation in $N=32$ with $N_{t}=16$ at low temperatures up to $T=3.5$.

Fig. 1 shows the internal energy normalized by $N^{2}$ against temperature. In the left figure, at high temperatures, lattice results for eq.(5.2) are coincident with the high temperature expansion at NLO, given by eq.(3.9). As temperature decreases, they are getting close to the expectation of the gravity side(dashed blue curve), given by eq.(5.1). Also, the right figure shows the low temperatures region of the left one. Results of $N=32$ are fairly close to the expectation, however, simulation temperatures seem to be still somewhat high to check the duality.

Values of the internal energy by this work are systematically smaller than those of the previous work done by Nishimura et al[4]. However, this difference does not immediately mean a serious contradiction between two works, because both were results obtained at the finite cut-off. To obtain determinate results, simulations for taking the continuum limit are currently running[12]. 


\section{Acknowledgments}

The calculations were performed by using the RIKEN Integrated Cluster of Clusters (RICC) facility. This work is supported by the Large Scale Simulation Program of High Energy Accelerator Research Organization (KEK).

\section{References}

[1] J. M. Maldacena, “The Large N limit of superconformal field theories and supergravity," Adv. Theor. Math. Phys. 2, 231 (1998) [hep-th/9711200].

[2] K. N. Anagnostopoulos, M. Hanada, J. Nishimura and S. Takeuchi, "Monte Carlo studies of supersymmetric matrix quantum mechanics with sixteen supercharges at finite temperature," Phys. Rev. Lett. 100, 021601 (2008) [arXiv:0707.4454 [hep-th]].

[3] M. Hanada, A. Miwa, J. Nishimura and S. Takeuchi, "Schwarzschild radius from Monte Carlo calculation of the Wilson loop in supersymmetric matrix quantum mechanics," Phys. Rev. Lett. 102, 181602 (2009) [arXiv:0811.2081 [hep-th]].

[4] M. Hanada, Y. Hyakutake, J. Nishimura and S. Takeuchi, "Higher derivative corrections to black hole thermodynamics from supersymmetric matrix quantum mechanics,” Phys. Rev. Lett. 102, 191602 (2009) [arXiv:0811.3102 [hep-th]].

[5] M. Hanada, J. Nishimura, Y. Sekino and T. Yoneya, "Monte Carlo studies of Matrix theory correlation functions,” Phys. Rev. Lett. 104, 151601 (2010) [arXiv:0911.1623 [hep-th]].

[6] S. Catterall and T. Wiseman, "Towards lattice simulation of the gauge theory duals to black holes and hot strings," JHEP 0712, 104 (2007) [arXiv:0706.3518 [hep-lat]]. "Black hole thermodynamics from simulations of lattice Yang-Mills theory," Phys. Rev. D 78, 041502 (2008) [arXiv:0803.4273 [hep-th]]. “Extracting black hole physics from the lattice," JHEP 1004, 077 (2010) [arXiv:0909.4947 [hep-th]].

[7] D. B. Kaplan and M. Unsal, "A Euclidean lattice construction of supersymmetric Yang-Mills theories with sixteen supercharges," JHEP 0509, 042 (2005) [hep-lat/0503039]. M. Unsal, "Supersymmetric deformations of type IIB matrix model as matrix regularization of N=4 SYM," JHEP 0604, 002

[8] F. Sugino, "A Lattice formulation of super Yang-Mills theories with exact supersymmetry," Nucl. Phys. Proc. Suppl. 140, 763 (2005) [Prog. Theor. Phys. Suppl. 164, 138 (2007)] [hep-lat/0409036]. "Various super Yang-Mills theories with exact supersymmetry on the lattice," JHEP 0501, 016 (2005) [hep-lat/0410035].

[9] N. Kawahara, J. Nishimura and S. Takeuchi, "High temperature expansion in supersymmetric matrix quantum mechanics,” JHEP 0712, 103 (2007) [arXiv:0710.2188 [hep-th]].

[10] I. R. Klebanov and A. A. Tseytlin, “Entropy of near extremal black p-branes,” Nucl. Phys. B 475, 164 (1996) [hep-th/9604089].

[11] D.Kadoh and S.Kamata, "Toward numerical verification of the gauge/gravity duality in 1d SYM with 16 supercharges.", in preparation.

[12] D.Kadoh and S.Kamata, "Black hole thermodynamics from lattice simulations of 1d SYM with 16 supercharges.", in preparation. 